Mar. Drugs 2007, 5, 1-5

Marine Drugs

ISSN 1660-3397

(C) 2007 by MDPI

www.mdpi.org/marinedrugs

Full Original Paper

\title{
Effects of Melophlins on Colony Formation of Chinese Hamster V79 Cells and IL-8 Production in PMA-stimulated HL-60 Cells
}

Taiko Oda $^{1, *}$, Ayako Fujita ${ }^{2}$, Jinzhong Xu ${ }^{2,3}$, Masataka Mochizuki ${ }^{1}$, Kazuyo Ukai ${ }^{3}$ and Michio $^{2}$ Namikoshi $^{3}$

1 Kyoritsu University of Pharmacy, Shibakoen, Minato-ku, Tokyo 105-8512, Japan

E-mail: oda-ti@kyoritsu-ph.ac.jp (T. O.). E-mail: mochizuki-mt@kyoritsu-ph.ac.jp (M. M.)

2 Department of Ocean Sciences, Tokyo University of Marine Science and Technology, Minato-ku,

Tokyo 108-8477, Japan

E-mail: K2006001@kyoritsu-ph.ac.jp (A. F.). E-mail: xujinzhong62jp@yahoo.co.jp (J. X.)

3 Tohoku Pharmaceutical University, Komatsushima, Aoba-ku, Sendai 981-8558, Japan

E-mail: ukai_k@tohoku-pharm.ac.jp (K. U.). E-mail: mnami@tohoku-pharm.ac.jp (M. N.)

* Author to whom correspondence should be addressed. Tel., +81-3-5400-2497; fax,

+81-3-5400-2497; e-mail: oda-ti@kyoritsu-ph.ac.jp

Received: 30 November 2006 / Accepted: 18 January 2007 / Published: 18 January 2007

\begin{abstract}
We have recently isolated four new melophlins P (1), Q (2), R (3), and S (4) together with seven known melophlins A (5), D (6), E (7), G (8), H (9), I (10), and O (11) from two marine sponges of the genus Melophlus collected in Palau. In this study, the influence of these compounds on the colony formation of Chinese hamster V79 cells and the production of IL-8 in PMA-stimulated HL-60 cells were examined. These 11 compounds did not show any effect on IL-8 production. The $\mathrm{EC}_{50}$ values of compounds 2, 3, 4, 5, 7, 9, 10, and 11 against V79 cells were 44.0, 13.3, 16.7, 27.2, 19.8, 8.5, 23.1, and 9.6 $\mu \mathrm{M}$, respectively. The linear-chain-type compounds $(\mathbf{1}, \mathbf{6}$, and $\mathbf{8})$ were not active against V79 cells at $50 \mu \mathrm{M}$. Although the growth inhibitory activity of these melophlins was not remarkable, some structure-activity relationships of these compounds against V79 and murine leukemia L1210 cells were observed.
\end{abstract}

Keywords: Melophlin, marine sponge, Melophlus sp., V79 cell, cell proliferation. 


\section{Introduction}

Marine sponges (Porifera) have been prolific sources of many interesting bioactive substances and are still an important resource for marine natural product chemistry. In the course of our studies on biologically active natural products of marine organisms, we have recently isolated four new melophlins P (1), Q (2), R (3), and S (4) together with seven known melophlins A (5), D (6), E (7), G (8), H (9), I (10), and O (11) from two marine sponges of the genus Melophlus collected in Palau [1]. Melophlins are tetramic acid derivatives possessing a long alkyl chain and have been isolated from Melophlus sarasinorum [1-3]. Compounds 1-11, except 8, showed weak to modest growth inhibitory activity against a murine leukemia cell line L1210 [1]. Melophlins A (5) and B (possessing the $\mathrm{C}_{11}$ alkyl chain and two methyl groups at C-5 $\alpha$ and C-9) were reported to have moderate cytotoxicity against HL-60 and arrest NIH3T3 fibroblasts in the G1 phase of the cell cycle [2]. Furthermore, melophlins A and B were found to reverse the morphology of H-ras-transformed HIH3T3 cells to normal and might act on the components of the ras-mediated signal transduction pathway [2].

Therefore, we intended to investigate the influence of 11 compounds (1-11) on the colony formation of Chinese hamster V79 cells. This bioassay reflects the direct action of compounds on the cells. We also observed the effect of 11 compounds (1-11) on the production of an inflammatory cytokine, IL-8, in PMA-stimulated HL-60 cells since the inhibition of cell proliferation sometimes correlates with IL-8 production. In this paper, we would like to describe some structure-activity relationships of these compounds against V79 and L1210 cells.
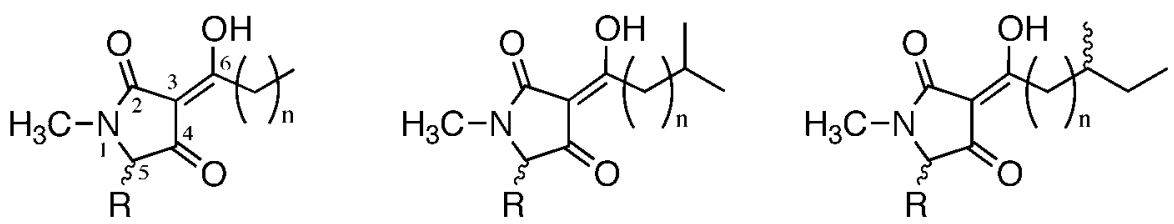

\begin{tabular}{cll} 
Melophlin & $\mathrm{R}$ & $\mathrm{n}$ \\
\hline $\mathrm{P} \mathrm{(1)}$ & $\mathrm{CH}_{3}$ & 14 \\
$\mathrm{~A} \mathrm{(5)}$ & $\mathrm{H}$ & 14 \\
$\mathrm{D} \mathrm{(6)}$ & $\mathrm{H}$ & 13 \\
$\mathrm{G} \mathrm{(8)}$ & $\mathrm{H}$ & 12
\end{tabular}
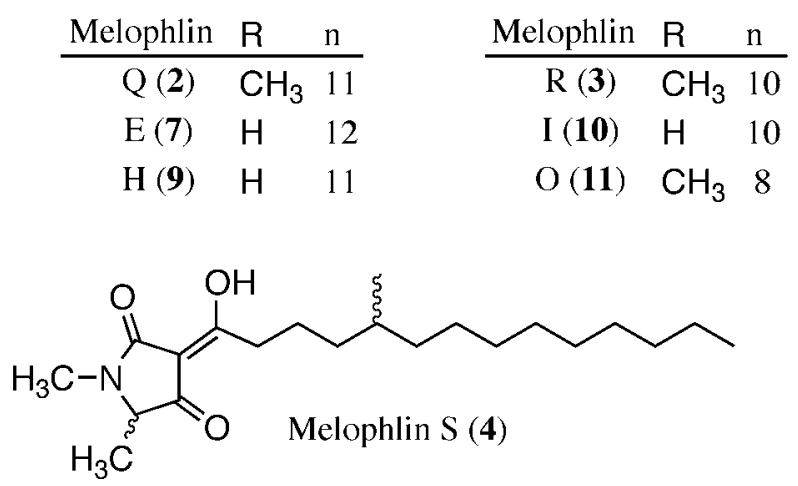

Figure 1. Structures of compounds 1-11.

\section{Materials and Methods}

\subsection{Materials}

Melophlins were isolated from two marine sponges of the genus Melophlus collected in Palau as described previously [1]. The structures of 11 compounds are shown in Figure 1. Dimethylsulfoxide 
(DMSO) was purchased from Pierce Chemical Co. (Rockfield, IL), and fetal bovine serum (FBS) was obtained from GIBCO after checking the lot. All other reagents and chemicals were of the highest grade available commercially.

\subsection{Cell lines and culture conditions}

Chinese hamster V79 cells were grown as a monolayer culture in Eagle's MEM (Nissui Seiyaku Co., Ltd., Tokyo, Japan) with 10\% heat-inactivated FBS. The HL-60 cell line was obtained from the Japanese Cancer Research Resources Bank (JCRB, Kamiyoga, Tokyo, Japan) and maintained in tissue culture dishes in RPMI 1640 medium (Nissui Seiyaku, Tokyo, Japan) supplemented with 10\% heat-inactivated FBS, $2 \mathrm{mM}$ glutamine, $100 \mathrm{U} / \mathrm{ml}$ of penicillin $\mathrm{G}$, and $100 \mu \mathrm{g} / \mathrm{ml}$ of streptomycin.

\subsection{Relative plating efficiency}

The relative plating efficiencies against V79 cells were determined as the ratio of the number of colonies in various concentrations of samples to that in the sample-free control, as described in previous papers [4,5]. Two hundred cells were seeded onto a 60/15-mm plastic plate with 4 ml culture medium and incubated overnight at $37^{\circ} \mathrm{C}$. After each sample in DMSO $(4 \mu \mathrm{l})$ was added to the culture medium, cells were further cultured for four days. The numbers of colonies in the sample plates were counted and compared with those in the control cultures.

\subsection{Detection of human IL-8 by ELISA}

The IL-8 concentrations of the culture supernatants under control and various test conditions were measured by ELISA using a combination of monoclonal and polyclonal antibodies [6]. All samples were assayed at least in duplicate. Data were analyzed as the mean \pm SE of three independent experiments.

\section{Results and Discussion}

Melophlin A (5) has been reported to inhibit the cell proliferation of HL-60 at the IC 50 value of 0.2 $\mu \mathrm{g} / \mathrm{ml}(0.57 \mu \mathrm{M})$ [2]. This compound arrested NIH3T3 fibroblast cells in the G1 phase of the cell cycle at $1 \mu \mathrm{g} / \mathrm{ml}(2.85 \mu \mathrm{M})$ and reversed the morphology of H-ras transformed NIH3T3 cells to normal at $5 \mu \mathrm{g} / \mathrm{ml}(14.2 \mu \mathrm{M})$ [2]. On the other hand, melophlins C, E (7), G (8), H (9), I (10), M, N, and O (11) were not cytotoxic to HL-60, HELA, or TF-1 cells [3]. In our experiment, compounds 1-7 and 9-11 showed weak to modest inhibitory activity against L1210 cells (Table 1) [1]; therefore, we examined the effect of compounds 1-11 on the rate of colony formation using V79 cells. This bioassay reflects the direct action of compounds on the cells. Since the production of an inflammatory cytokine, IL-8, is, in some cases, correlated with the inhibition of cell proliferation, we also observed the influence of 11 compounds on the IL-8 production system mediated by PMA-stimulated HL-60 cells.

Although the inhibitory activities of the 11 compounds on the colony formation of V79 were not remarkable, melophlins $\mathrm{H}(\mathbf{9})$ and $\mathrm{O}(\mathbf{1 1})$ showed activity at $\mathrm{EC}_{50}$ values of 8.5 and $9.6 \mu \mathrm{M}$, 
respectively (Table 1); however, compounds 1-11 did not affect the production of IL-8.

Table 1. Growth inhibitory activities of compounds 1-11 against Chinese hamster V79 and murine leukemia L1210 cells.

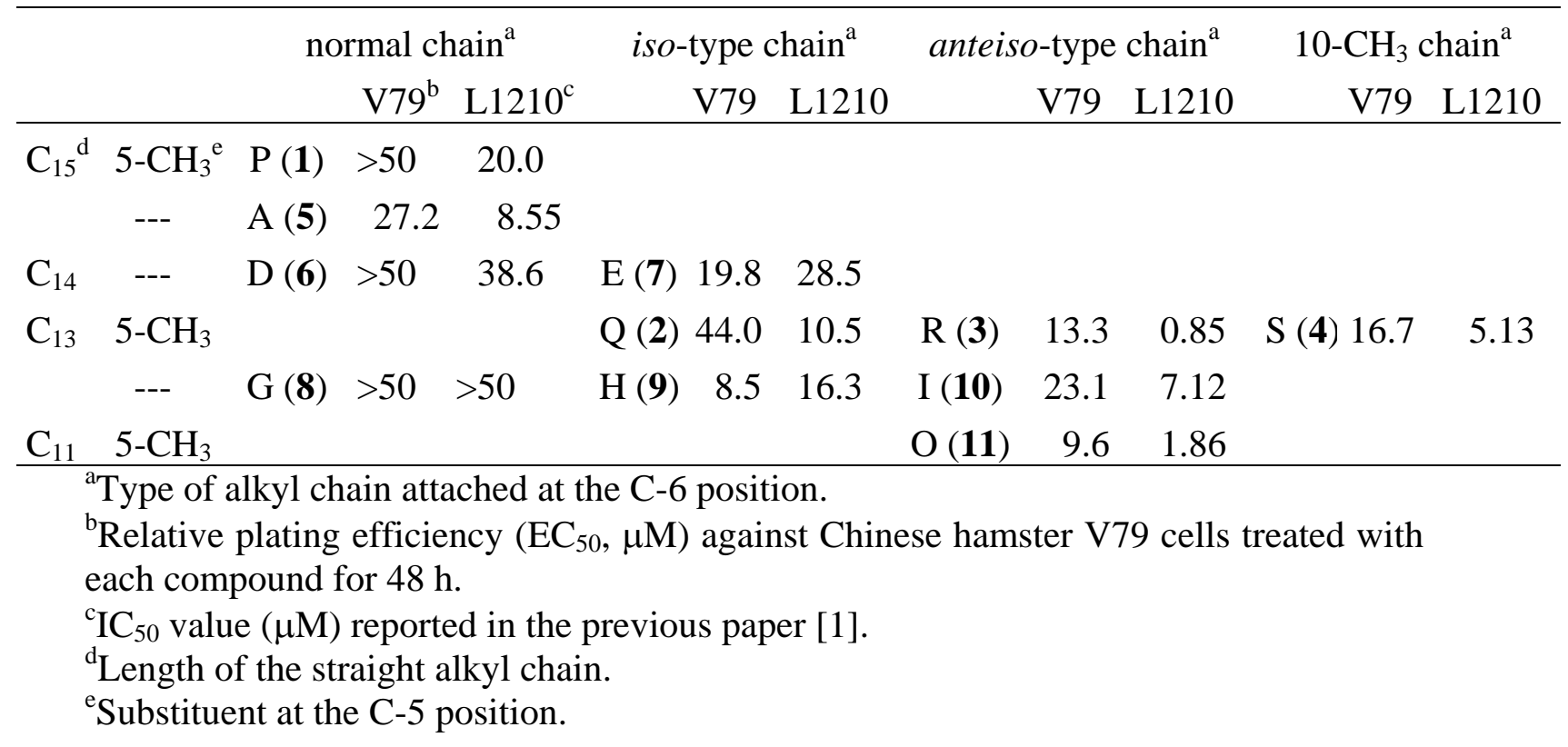

Linear-chain-type compounds (1, 5, 6, and 8) were least active among the four chain types (Table 1). The methyl group at C-5 reduced the activity of linear-chain-type and iso-type compounds (2, 7, and 9), while the activity of anteiso-type compounds (3, 10, and 11) were increased by C-5 methylation. Activity was decreased as the chain length increased for linear-chain-type compounds. On the other hand, activity was increased as the chain length decreased for iso-type and anteiso-type compounds.

These structure-activity relationships were detected against both V79 and L1210 cells except for the iso-type compound with the C-5 methyl group (compound 2), which showed stronger activity against L1210 cells than compound 9. The activity of the 10-methyl compound (4) against V79 and L1210 cells was stronger than that of the iso-type compound (2) and weaker than that of the anteiso-type compound (3).

Therefore, it will be interesting to examine the in vitro growth inhibitory activity of compounds possessing iso-type or anteiso-type chains with a chain-length of less than $\mathrm{C}_{12}$ against human solid tumor cell lines.

\section{Acknowledgements}

We thank Dr. T. Kasahara of Kyoritsu University of Pharmacy for providing the IL-8 monoclonal and polyclonal antibodies and Mr. K. Nagata, Mr. Y. Tanaka, Ms. Y. Kojima, and Ms. I. Kusano for their excellent technical assistance. This work was supported in part by a Grant-in-Aid for Scientific Research on Priority Areas 17035029 from The Ministry of Education, Culture, Sports, Science and Technology (MEXT) of Japan to M. N. 


\section{References}

1. Xu, J.; Hasegawa, M.; Harada, K.-I.; Kobayashi, H.; Nagai, H.; Namikoshi, M. Melophlins P, Q, $\mathrm{R}$, and S: four new tetramic acid derivatives, from two Palauan marine sponges of the genus Melophlus. Chem. Pharm. Bull. 2006, 54, 852-854.

2. Aoki, S.; Higuchi, K.; Ye, Y.; Satari, R.; Kobayashi, M. Melophlins A and B, novel tetramic acids reversing the phenotype of ras-transformed cells, from the marine sponge Melophlus sarasinorum. Tetrahedron 2000, 56, 1833-1836.

3. Wang, C.-Y.; Wang, B.-G.; Wiryowidagdo, S.; Wray, V.; van Soest, R.; Steube, K. G.; Guan, H.-S.; Proksch, P.; Ebel, R. Melophlins C-O, thirteen novel tetramic acids from the marine sponge Melophlus sarasinorum. J. Nat. Prod. 2003, 66, 51-56.

4. Sakakibara, Y.; Saito, I.; Ichinoseki, K.; Oda, T.; Kaneko, M.; Saito, H.; Kodama, M.; Sato, Y. Effects of diethylstilbestrol and its methyl ethers on aneuploidy induction and microtubule distribution in Chinese hamster V79 cells Mutat. Res. 1991, 263, 269-76.

5. Sato, Y.; Sakakibara, Y.; Oda, T.; Aizu-Yokota, E.; Ichinoseki, I. Effects of estradiol and ethynylestradiol on microtubule distribution in Chinese hamster V79 cells. Chem. Pharm. Bull. 1992, 40, 182-184.

6. Kasahara, T.; Oda, T.; Hatake, K.; Akiyama, M.; Mukaida, N.; Matsushima, K. Interleukin-8 and monocyte chemotactic protein-1 production by a human glioblastoma cell line, T98G in coculture with monocytes: involvement of monocyte-derived interleukin-1alpha. Eur. Cytokine Netw. 1998, 9, 47-55.

Samples Availability: Not available.

(C) 2007 by MDPI (http://www.mdpi.org). Reproduction is permitted for noncommercial purposes. 